Clemson University

TigerPrints

Publications

Bioengineering

8-2018

Mechanically assisted electrochemical degradation of aluminaTiC composites

Hetal Umesh Maharaja

Guigen Zhang

Follow this and additional works at: https://tigerprints.clemson.edu/bioengineering_pubs

Part of the Biomedical Engineering and Bioengineering Commons, and the Computer Engineering Commons 


\title{
Mechanically assisted electrochemical degradation of alumina-TiC composites
}

\author{
Hetal U Maharaja ${ }^{1,3}$ and Guigen Zhang ${ }^{1,2,3}$ \\ ${ }^{1}$ Department of Bioengineering, Clemson University, 301 Rhodes Research Center, \\ Clemson, SC 29634-0905, USA \\ ${ }^{2}$ Department of Electrical and Computer Engineering, Clemson University, 301 Rhodes \\ Research Center, Clemson, SC 29634-0905, USA \\ ${ }^{3}$ Institute for Biological Interfaces of Engineering, Clemson University, 401 Rhodes \\ Research Center, Clemson, SC 29634-0905, USA
}

Corresponding author (current address): Guigen Zhang, F. Joseph Halcomb III, M.D. Department of Biomedical Engineering, University of Kentucky, 522A RMB, 143 Graham Avenue, Lexington, KY 40506-0108; guigen.bme@uky.edu

\begin{abstract}
Alumina-TiC composite material is a tough ceramic composite with excellent hardness, wear resistance and oxidation resistance in dry and hightemperature conditions. In aqueous conditions, however, it is likely to be electrochemically active facilitating charge transfer processes due to the conductive nature of TiC. For application as an orthopedic biomaterial, it is crucial to assess the electrochemical behavior of this composite, especially under a combined mechanical and electrochemical environment. In this study, we examined the mechanically assisted electrochemical performance of alumina-TiC composite in an aqueous environment. The spontaneous electrochemical response to brushing abrasion were measured. Changes in the magnitude of electrochemical current with abrasion test conditions and possible causal relationship to the alteration in surface morphology were examined. Results showed that the alumina matrix underwent abrasive wear with evidence of microploughing and grain boundary damage. Chemical analysis revealed $\mathrm{TiO}_{2}$ formation in the abraded region, indicating oxidation of the conductive TiC domain. Furthermore, wear debris from alumina abrasion appeared to affect reaction kinetics at the composite-electrolyte interface. From this work, we established that the composite undergoes abrasion assisted electrochemical degradation even in gentle abrasive conditions and the severity of degradation is related to temperature and conditions of test environment.
\end{abstract}


Keywords: Alumina-TiC, ceramic composite, abrasion, low load, oxidation, electrochemical, brushing, mechanically assisted electrochemical degradation, oxidative wear, microploughing

\section{Introduction}

Metal alloys such as Ti-6Al-4V and CoCrMo, known for their biocompatibility and mechanical strength, are common biomaterials for total hip replacements (THR) [1]. The surface of these metallic biomaterials often forms a passive oxide film providing resistance against corrosion or electrochemical degradation. Electrochemical degradation is a charge-transfer process in which metallic materials react and/or interact with the aqueous environment. Such a process always includes oxidation of metallic components and the concomitant reduction of active species in the aqueous environment somewhere else on the implant surface. Whether an electrochemical reaction will occur or not under certain given conditions is governed by its thermodynamic favorability (Gibbs free energy of reaction, $\Delta G$ ) which is a function of the type and state of reactants, surface property, reaction kinetics, chemical species and their concentration, and temperature, among others [2].

The passive oxide film on metal alloys is typically a few nanometers thick and provides protection against the dissolution of metals into metal ions and electrons when exposed to aqueous environments. High mechanical loads and relative motion between two contacting surfaces of articulating load-bearing joints could cause disruption of this oxide film that exposes the underlying metal alloy resulting in oxidation and the release of metal ions into the surrounding synovial fluid $[3,4,5]$. The released metal ions can possibly illicit inflammatory responses, leading to osteolysis and eventual aseptic implant loosening from failure of the osteointegration process $[6,7]$.

Mechanically assisted electrochemical degradation is an electrochemical process triggered by a mechanical condition such as abrasion, micromotion or fretting between two articulating surfaces, leading to damage of the protective oxide film. The undesirable electrochemical activity of the biomaterial may be compounded by the release of wear debris that can further damage the protective oxide film and compromise the integrity of the implant surface. In tribology terms, it is often regarded as a tribo-electrochemical wear process.

Ceramic biomaterials are known to provide superior mechanical strength, wear resistance, hardness, chemical inertness than their metallic counterparts [8,9]. For example, alumina-based ceramic composites demonstrate reduced friction as articulating joints and improved wear resistance compared with metal-metal or metal-polymer combinations [10]. With a Vicker's hardness of more than 2000, alumina $\left(\mathrm{Al}_{2} \mathrm{O}_{3}\right)$ can be polished to a smooth surface. It possesses high wettability providing better adhesion to lubricating fluid, enabling it to have a much lower wear rate as articulating components, some $0.025 \mu \mathrm{m} / \mathrm{yr}$ to $4 \mu \mathrm{m} / \mathrm{yr}$ in comparison with 
$100 \mu \mathrm{m} / \mathrm{yr}$ for metal-polyethylene articulating pairs [1]. Alumina is also one of the most thermodynamically stable oxides of aluminum and is less susceptible to degradation by usual oxidation making it highly biocompatible [11].

There have been many advances in alumina manufacturing processes since the 1970s. Today alumina used for total hip arthroplasties has fewer impurities, smaller grain sizes, higher density and improved fracture toughness (by the addition of zirconia and other oxides) [10, 12]. However, due to their inherent brittleness, ceramics are not able to sustain high impact or non-uniform loads [1] and show little to no plastic deformation under extreme mechanical situations, and they are prone to micro-fracture under abrasive conditions. The resulting fragments or debris, even if not harmful to the host tissues, could act as third body particles to accelerate wear damage. Some studies have shown prominent inflammatory responses to ceramic wear debris $[13,14]$ requiring revision surgeries due to aseptic loosening for ceramic-on-ceramic hip prosthesis [15].

The intrinsic brittleness of alumina can be reduced by the addition of hard reinforcements like metal carbides, nitrides and oxides. Monolithic alumina when enhanced by metal refractory ceramic reinforcements like titanium carbide (TiC), titanium carbonitride $(\mathrm{Ti}(\mathrm{C}, \mathrm{N}))$, tungsten-titanium composite carbide $((\mathrm{W}, \mathrm{Ti}) \mathrm{C})$ yield a composite with increased flexural strength, fracture toughness, hardness and an improved friction coefficient $[16,17,18]$. The alumina- $\mathrm{TiO}_{2}$ nanocomposite exhibited lower wear volume and better mechanical properties with a $10 \mathrm{~mol} \% \mathrm{TiO}_{2}$ addition [19]. Likewise, mechanical properties and wear behavior of alumina-TiN as a potential biomaterial has been explored too [8]. Titanium carbide ( $\mathrm{TiC})$ added to alumina increases hardness, toughness and more importantly wear resistance of just plain alumina $[18,20]$. Due to superior hardness, TiC coatings on titanium substrates enhance resistance to tribochemical wear and offer corrosion resistance to an underlying metal substrate. It also improves osseointegration by stimulating the growth of osteoblasts and their proliferation, offering a biocompatible interface to metallic or polymer substrates [21].

Besides improvement in mechanical and wear properties of alumina by the transition metal carbides and nitrides, it is important to consider the electrochemical behavior of a reinforcing material when evaluating chemical and tribological stability of an alumina composite. Even alumina, although an insulator [22], does not participate in the electrochemical processes, studies have shown that its wear resistance decreases in an aqueous environment due to its hydrophilic nature by reacting with water to form aluminum hydroxide in basic and acidic environments at elevated temperatures [23,24]. Moreover, these transition metal refractory ceramics mentioned earlier demonstrate metal-like conductivity, enabling charge transfer during electrochemical reactions [25-28], hence electrical and chemical implications of their additions to alumina need to be carefully assessed.

Besides its extreme hardness, TiC is a conductive ceramic with resistivity of $0.003-0.008 \Omega-\mathrm{m}$ (vs $\mathrm{Cu}-1.72 \times 10^{-8} \Omega-\mathrm{m}$ ) and can partake in electrochemical processes in a chemical environment [22]. TiC, when used as reinforcement for alumina to improve wear resistance, retains its conductive nature [20] and this property is in fact favorable in magnetic recording disk drive application where the 
metal-like conductivity of $\mathrm{TiC}$ is beneficial to dissipate charge build up due to frictional contact [29]. Electrochemical nature of TiC was highlighted in studies $[30,31]$ that have shown anodic dissolution of $\mathrm{TiC}$ in aggressive chemical conditions and [32] where $\mathrm{TiC}$ nanowires exhibited enhanced electrocatalytic properties allowing facile electron transfer and redox activity. It is known from a study [29] that alumina-TiC composites have high oxidation resistance in air and nitrogen environments for temperatures up to $350^{\circ} \mathrm{C}$. However, alumina-TiC composites also undergo oxidative wear in dry conditions. Bare alumina-TiC has been observed to release $\mathrm{CO}_{2}$ as a byproduct of tribochemical wear of $\mathrm{TiC}$ at 120 ${ }^{\circ} \mathrm{C}$ under dry sliding wear [33] and that oxygen chemisorption and carbon oxidation is catalyzed by alumina-TiC. Such a tribochemical wear mechanism is likely to be enhanced under an aqueous environment that allows for continuous electrochemical interactions.

Considering improved mechanical strength, wear resistance of alumina-TiC and biocompatibility of $\mathrm{TiC}$ itself, alumina-TiC composites may be regarded as an appealing biomaterial for load bearing implants. However, the tendency of $\mathrm{TiC}$ to facilitate charge transfer in electrochemical processes and reactivity of alumina in a wet environment pose a need to evaluate the electrochemical behavior of alumina$\mathrm{TiC}$ composites. Hence the electrochemical activity of alumina-TiC in an aqueous chemical environment, especially when compounded by destructive mechanical processes like high impact loads, abrasion, cyclic fatigue, frictional wear, needs to be evaluated. Such an evaluation would enable a better material design of the ceramic composite for biomedical applications.

In this study, we focus our efforts on investigating the effect of $\mathrm{TiC}$ reinforcement on the electrochemical degradation of alumina-TiC composites. We aim to elucidate the degradation mechanism involved by studying the electrochemical response of alumina-TiC composites when it is abraded in an aqueous environment and assess corresponding alterations in surface chemistry and appearance with quantitative and qualitative techniques, respectively. The experimental methods employed to understand the interplay of mechanical and electrochemical processes are as follows:

Table 1-Experimental methods used in the study

\begin{tabular}{|c|c|}
\hline & \multicolumn{1}{|c|}{ Tests } \\
\hline Quantitative/Semi-quantitative & i) Electrochemical Methods: \\
& a) Open circuit current (ZRA) \\
& b) Electrochemical Impedance \\
Spectroscopy \\
\hline & ii) Chemical analysis: \\
& a) Inductively coupled plasma \\
& mass spectrometry (ICP-MS) \\
& b) Xray Photoelectron \\
& Spectroscopy (XPS) \\
\hline
\end{tabular}




\begin{tabular}{|l|l|}
\hline Qualitative & $\begin{array}{l}\text { Scanning Electron Microscopy } \\
(\text { SEM })\end{array}$ \\
\hline
\end{tabular}

\section{Methods and Materials}

To examine the effect of gentle abrasion on the electrochemical behavior of alumina-TiC composites, we built an experimental apparatus allowing us to abrade the composite with a brush in an aqueous environment while simultaneously measuring electrochemical response from the composite. Doing so will enable us to study the spontaneous oxidative processes induced by brushing abrasion and establish interdependence among different parameters of abrasion, including temperature, brushing acceleration and speed, electrochemical potential and current. Further characterization of the degradation of alumina composites by brushing abrasion is achieved by comparing microstructural damage due to abrasion in a dry environment.

\subsection{Brushing abrasion setup}

The setup built for the brushing tests is shown in Fig.1. Its center piece is a motorized overhead stirrer (Eurostar power control-visc, IKA Works, Wilmington, NC) for rotational-motion of brush about the vertical axis. Brushing abrasion of an alumina-TiC composite sample is accomplished with a nylon brush, attached to the end of the motor shaft. The acceleration and speed of the stirrer motor is controlled using the labworldsoft 5 program (IKA Works, Wilmington, NC). This setup also simultaneously measures the electrochemical response of alumina-TiC composites to brushing abrasion with a potentiostat (VersaStat MC, Princeton Applied Research, Oak Ridge, TN). A heating unit in the set up comprises of a J-type thermocouple, heating tape (BIH051020L, BriskHeat, Columbus, OH) and a digital temperature controller ITC-106 (Inkbird, Shenzhen, China) is used to control the temperature of the test solution (electrolyte) throughout the duration of a test. The contact load between the brush and the composite sample is monitored and controlled using a force sensor (flexiforce sensor: A201, Tekscan, South Boston, MA). In a typical brushing test, a nylon brush is brought into contact with the surface of the alumina-TiC composite sample and a contact force of $45 \mathrm{gm}-\mathrm{f}$ ( 0.44 $\mathrm{N})$ is achieved by the force monitoring unit and by adjusting the base plate on linear translation stage. This force may fluctuate about this constant set value during brushing due to scattered contact of rotating bristles of the brush. A ramp scheme 
is designed in the labworldsoft to control the acceleration of the motor from rest to a preset maximum brushing speed, governing the rate and degree of abrasion.

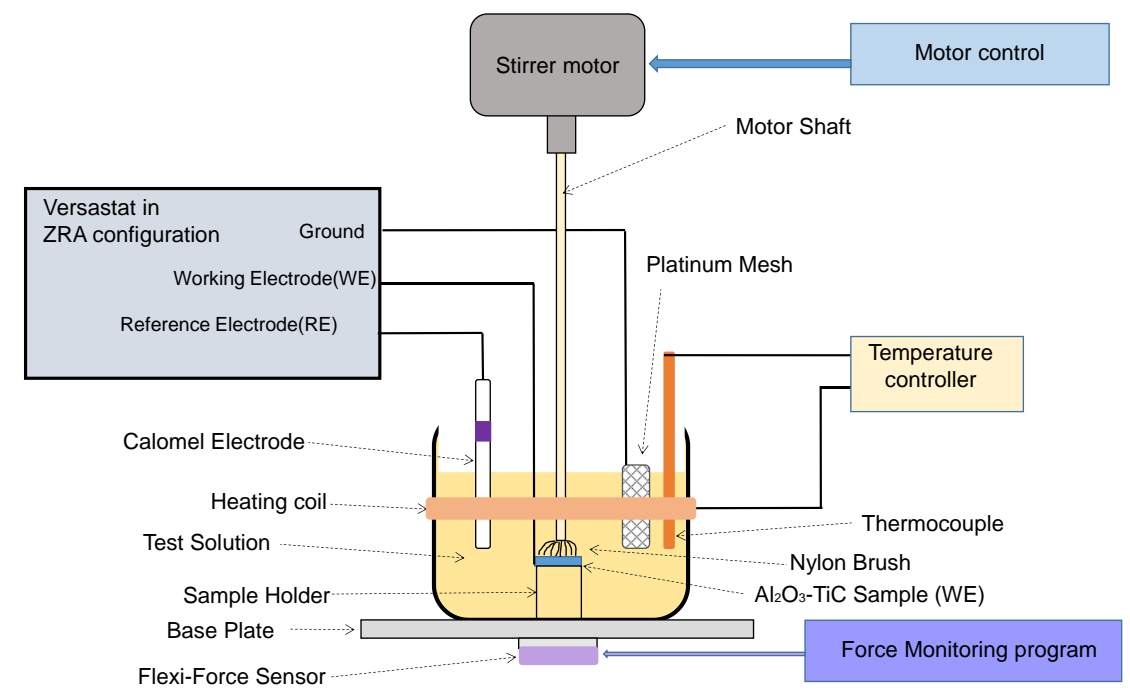

Fig. 1. Experiment apparatus set up for brushing abrasion

\subsection{Sample preparation}

For every test, commercially available alumina-TiC (70\%/30\%) samples, $10 \times 10$ $\mathrm{mm}$ in size, were first cleaned ultrasonically for 10 minutes in ethanol followed by rinsing in deionized (DI) water. An electrical connection with the composite sample was established using a copper tape and the test sample was used as the working electrode (WE). All sides of the sample except the top surface and copper tape to be exposed to the test solution were coated with lacquer to minimize unwanted interferences from copper. The prepared sample was kept at a fixed position on a sample holder with its top surface facing up. A nylon brush in the form of a bundle of bristles with a polyethylene base was used for brushing about the vertical axis. The nylon brush was cleaned by sonicating in ethanol for 10 minutes followed by rinsing in DI water.

\subsection{Electrochemical measurements}


Electrochemical measurements were made using a VersaStat MC. A Saturated Calomel Electrode (SCE) was used as the reference electrode (RE), a platinum mesh as the counter electrode for potentiostatic (applied potential) and potentiodynamic tests and as a ground lead (GND) for open circuit condition (free potential) measurements. Micro90 ( $\mathrm{pH}$ \%.5), a corrosive organic solution diluted to $0.3 \%$ ( vol $/ \mathrm{vol})$ in DI water and heated to $75^{\circ} \mathrm{C}$, was used as an electrolyte unless stated otherwise. To monitor the brushing induced electrochemical current response without applying any electrical potential (free potential), a potentiostat set in a Zero Resistance Ammeter (ZRA) configuration was used in a three-electrode setting.

\subsection{Brushing abrasion testing}

Before starting the brushing abrasion, the open circuit potential (OCP) of the alumina-TiC sample was allowed to stabilize for 10 minutes while recording the electrochemical current. Brushing acceleration and speeds were controlled by adjusting the ramping times for the stirrer motor to reach maximum abrasion speed from rest. Following the brushing test, the composite sample was rinsed with DI water and ethanol and stored for surface analysis.

By using the same test parameters and setup as described above for every run of an experiment, a systematic study of brushing abrasion was performed to characterize the mechanically assisted degradation of alumina-TiC composites and identify factors that affect the degradation process.

\subsubsection{Effect of brushing acceleration and speed}

Since the ramping time to reach maximum speed controls the amount of rotational force imparted to the surface features under abrasion, the effect of ramping time was studied under three different ramping schemes: 10, 40 and 70 seconds to ramp the rotational speed of brush from rest to maximum speed of 800 revolutions per minute (rpm). Total test duration was about 17 minutes with 10 minutes for OCP stabilization, 5 minutes of brushing, and some remaining time for motion actuation and slowdown. Two maximum brushing speeds were used: 500 and $1200 \mathrm{rpm}$. After the brushing tests, the resulting surfaces were imaged and analyzed for morphological changes. This set of experiment was designed to correlate electrochemical responses with the abrasion ramping time and speed.

\subsubsection{Effect of temperature}


Temperature is an important factor that governs the thermodynamic favorability of electrochemical processes, rate of reaction and conductivity of the test solution. To understand the effect of temperature, the prolonged exposure of alumina-TiC composites to an aqueous alkaline environment was carried out in a heated $\left(75^{\circ} \mathrm{C}\right)$ and room $\left(25^{\circ} \mathrm{C}\right)$ temperature. The total duration of these tests was two hours with brushing abrasion for 6 minutes (in three consecutive brushing cycles of 2 minutes each). The brushing abrasion parameters were kept the same: a contact force of 45 grams, ramping time of $10 \mathrm{~s}$, and maximum brushing speed of $800 \mathrm{rpm}$.

\subsubsection{Effect of environment}

To ascertain if the material degradation mechanism is an abrasion assisted electrochemical process and not just a tribological process, brushing abrasion in dry conditions (no electrolyte) was also performed. The resulting microstructural damage of the dry-test samples was examined and compared with the wet-test samples. Aside from the dry and wet difference, other experimental settings were kept unchanged at a contact force of 45 grams, ramping time at $10 \mathrm{~s}$, and maximum brushing speed of $800 \mathrm{rpm}$. In this way, the surface damage incurred would be mainly due to brushing abrasion because the electrochemical interactions of $\mathrm{TiC}$ with the aqueous environment were eliminated in dry abrasion.

\subsection{Electrochemical impedance study}

Electrochemical impedance spectroscopy (EIS) tests were undertaken using the same setup described earlier to characterize oxide formation and change of sampleelectrolyte interface properties. Impedance scans were taken before and after potentiostatic conditions (anodic and cathodic) and abrasion tests in Micro90 at $75^{\circ} \mathrm{C}$. Potentiostatic tests were performed to verify the propensity and stability of the oxide formation particularly on the $\mathrm{TiC}$ domain as alumina does not participate in the charge transfer processes. The value for the applied potential in potentiostatic tests was chosen from active cathodic and anodic regions of potentiodynamic tests which coincided with average OCP values (-250 mV vs SCE) observed in free potential mode during brushing abrasion. The experimental design for EIS analysis is shown in Table 2 below.

Table 2 - Experimental conditions for EIS study

\begin{tabular}{|l|c|c|c|l|}
\hline Experiment & Duration & Temperature & $\begin{array}{l}\text { Brushing } \\
\text { Abrasion }\end{array}$ & $\begin{array}{l}\text { Applied } \\
\text { Potential } \\
\text { vs SCE }\end{array}$ \\
\hline Anodic Biasing & 43 minutes & $75^{\circ} \mathrm{C}$ & No & $250 \mathrm{mV}$ \\
\hline Cathodic Biasing & 43 minutes & $75^{\circ} \mathrm{C}$ & No & $-250 \mathrm{mV}$ \\
\hline
\end{tabular}




\begin{tabular}{|l|c|c|c|c|}
\hline $\begin{array}{l}\text { No Brushing-No } \\
\text { Biasing }\end{array}$ & 43 minutes & $75^{\circ} \mathrm{C}$ & No & No \\
\hline Brushing-No Biasing & $\begin{array}{c}10 \text { minutes } \\
\text { OCP } \\
\text { stabilization } \\
+30 \text { minutes } \\
\text { brushing }+3 \\
\text { minutes to } \\
\text { restore }\end{array}$ & $75^{\circ} \mathrm{C}$ & Yes & No \\
\hline
\end{tabular}

Three runs of impedance test were performed before and after the application of each experimental condition listed in Table 2 to study the altered sample-electrolyte interface. In every impedance scan, alternating current (AC) perturbation with magnitude of $50 \mathrm{mV}$ was applied at open circuit condition in the frequency range of $1 \mathrm{~Hz}-50 \mathrm{kHz}$. For brushing abrasion experiments, 10s of ramping time with a maximum speed of $800 \mathrm{rpm}$ was applied after 10 minutes of OCP stabilization. Charge transfer resistance $\left(\mathrm{R}_{\mathrm{ct}}\right)$, the parameter of interest in this experimental design, was obtained with ZView by fitting an equivalent circuit model to the Nyquist plot acquired. Any alterations in charge transfer resistance before and after a test condition would give us clues about possible changes in oxide film on the $\mathrm{TiC}$ domain.

\subsection{Surface characterization}

Abrasive alterations in surface morphology were examined through comparison of images obtained from Scanning Electron Microscopy (SEM) (SU6600 and S4800, Hitachi High Technologies, Tokyo, Japan) for abraded samples and pristine samples. Changes caused by different parameters of brushing abrasion tests were analyzed. Note that with less energetic secondary electrons reflected from sample surfaces, images taken at low $\mathrm{kV}(0.7 \mathrm{kV})$ will reveal more superficial information than $5 \mathrm{kV}$, and that at the lower $\mathrm{kV}$, the contrast will be reversed and alumina matrix domain will appear as darker regions under $0.7 \mathrm{kV}$ rather than lighter under $5 \mathrm{kV}$.

\subsection{Chemical analysis}

As the mode of abrasion employed in this study is of gentle nature, to obtain measurable alterations in surface chemistry, a much longer duration of brushing abrasion was employed. To accelerate the surface chemistry changes by brushing abrasion, continuous brushing in heated Micro90 at $1000 \mathrm{rpm}$ (ramping time of 10 
s) for 2.5 hours was performed following 10 minutes of OCP stabilization. Abraded regions were marked under optical microscope for chemical analysis by X-ray Photoelectron Spectroscopy (XPS). Elemental scans for titanium were performed to observe changes in oxidation states after brushing abrasion. Atomic percentages of each element (Ti, C, Al and O) from XPS scans were obtained to gain a preliminary understanding of changes in surface chemistry due to the electrochemical process activated by brushing abrasion of alumina-TiC.

The test solution after brushing abrasion was also analyzed for any traces of titanium oxide or alumina particles released as debris during the prolonged brushing. ICP-MS (Inductively Coupled Plasma Mass Spectrometry) was employed to determine its elemental titanium and aluminum concentration. Untested Micro90 solution was also analyzed as a control.

\section{Results and Discussion}

\subsection{Electrochemical response to brushing abrasion}

Under OCP conditions, the composite shows a baseline current of nearly $1 \mu \mathrm{A}$ in the heated Micro90 environment, suggesting a dynamic electrochemical process on the sample surface. These measurable electrochemical interactions signify that the electro-active domain of the $\mathrm{TiC}$ composite during the OCP stabilizing period interacts with the test solution. After OCP stabilization, brushing abrasion results in typical 'passive layer breakdown' behavior as evident in both the OCP and current responses which are commonly observed with metal and metal alloys [3, 34, 35] as well as metal-ceramic composites [36]. In response to abrasion after OCP stabilization, electrochemical current shows a sharp increase with a concurrent negative drop in OCP as shown in Fig. 2, indicating activation of oxidative reactions on TiC. The decay of both current and OCP suggests a re-passivation process occurring to remedy the disruption of the oxide barrier on the sample surface. This re-passivation behavior may not completely form a compact non-porous film due to continuing abrasion when the brushing motion is going on. 


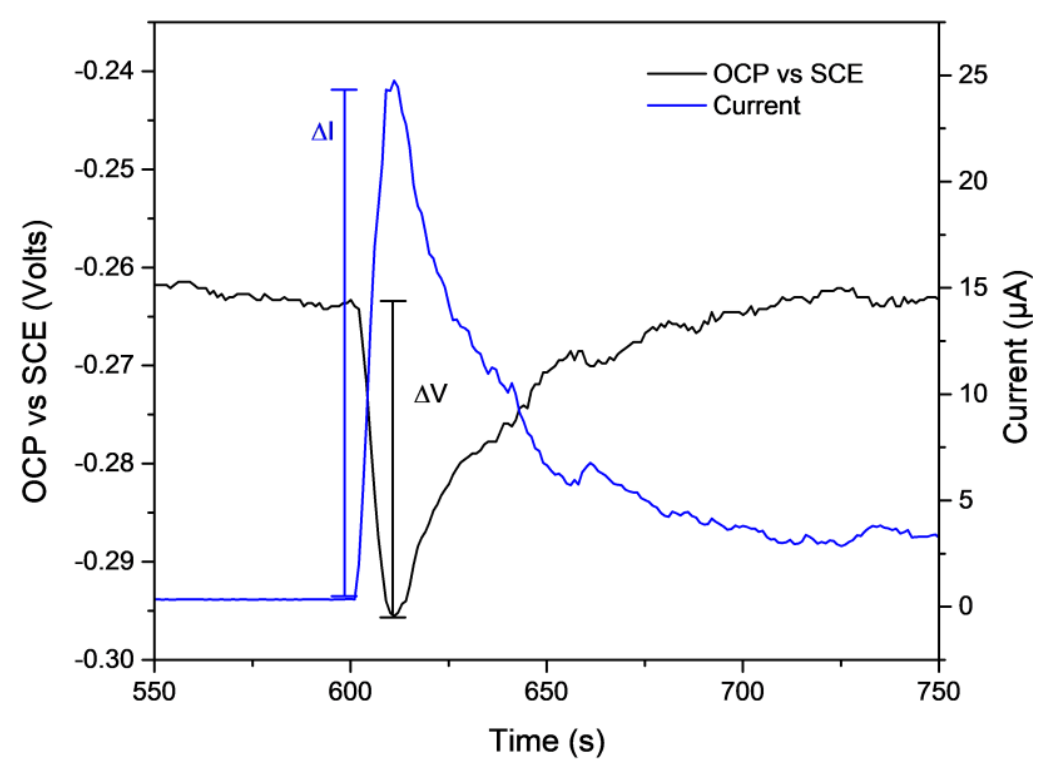

Fig. 2. Typical electrochemical response of alumina-TiC to brushing abrasion in Micro 90 at $75^{\circ} \mathrm{C}$

As the brushing stops, the current decays to its original rate of stabilization. Such behavior shows that the rate of electrochemical reaction on the $\mathrm{TiC}$ domain is increased due to brushing abrasion. Parameters like $\Delta \mathrm{V}$ and $\Delta \mathrm{I}$ depicted in Fig. 2 are the differential values of $\mathrm{OCP}$ and electrochemical current respectively from baseline, that provide a quantitative measure of the electrochemical activity of alumina-TiC initiated by abrasion.

Magnitudes of the electrochemical current and potential vary with brushing abrasion parameters like ramping speed and maximum brushing speed. As seen in Fig. 3a, current response to brushing with a ramping time of $10 \mathrm{~s}$ is the highest with the greatest average $\Delta \mathrm{V}(0.022 \mathrm{~V})$ and $\Delta \mathrm{I}(17.3 \mu \mathrm{A})$ as plotted in Fig.3b. Clearly, a shorter ramping time generates a larger rotational acceleration hence exerting greater abrasive forces on a sample surface than a longer ramping time, leading to more morphological damage. Similarly, a larger maximum speed generates a higher current and causes more surface damage. For example, as seen in Fig. 4a and Fig.4b, the case of $1200 \mathrm{rpm}$ maximum speed results in higher delta values for OCP and electrochemical current $(\Delta \mathrm{V}=0.035 \mathrm{~V}, \Delta \mathrm{I}=26.19 \mu \mathrm{A})$ than the case of $500 \mathrm{rpm}$ $(\Delta \mathrm{V}=0.017 \mathrm{~V}, \Delta \mathrm{I}=17.92 \mu \mathrm{A})$. These facts suggest that the abrasion of alumina-TiC activates an oxidative electrochemical process, resulting in increased chemical interactions with an aqueous environment and the corresponding electrochemical response depends on abrasion parameters. 
a)

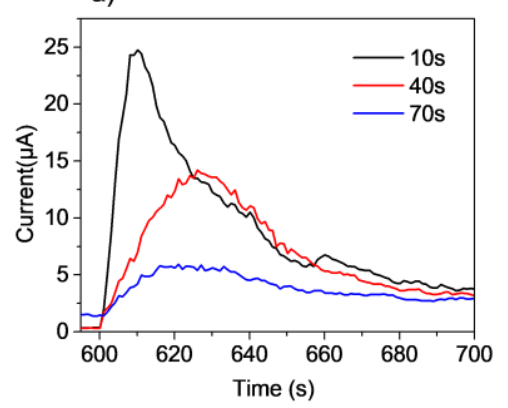

b)

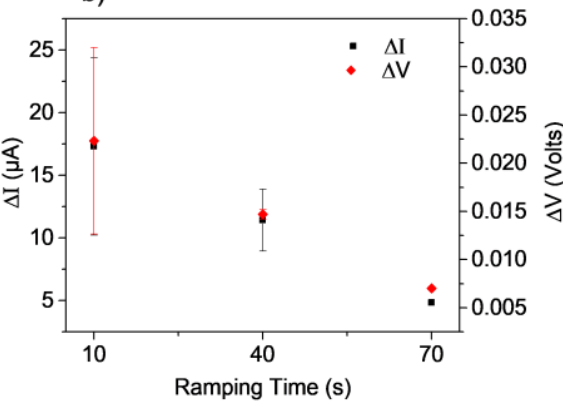

Fig. 3. Effect of ramping time on a) Current response to brushing abrasion at $10 \mathrm{~s}, 40 \mathrm{~s}, 70 \mathrm{~s}$ and b) Average $\Delta V$ and $\Delta I$ values for $n=3$ in Micro90 at $75^{\circ} \mathrm{C}$.

a)

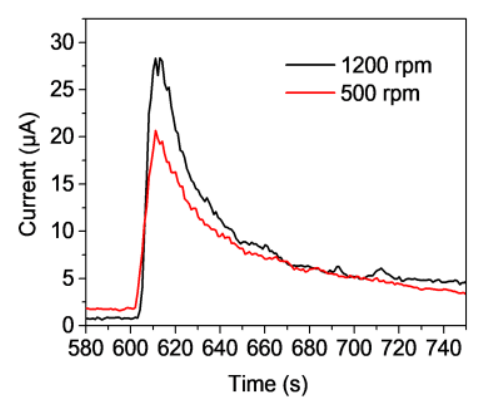

b)

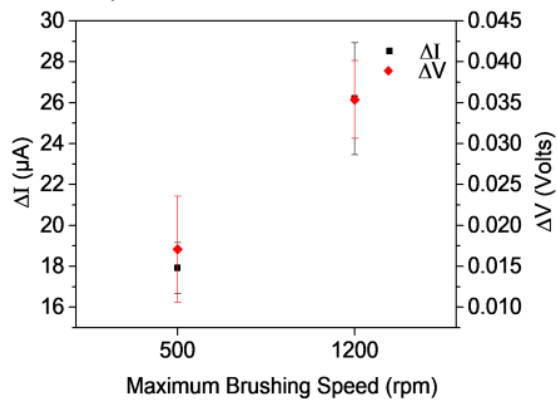

Fig. 4. Effect of maximum brushing speed on a) Current response to abrasion at $1200 \mathrm{rpm}$ and $500 \mathrm{rpm}$ and b) Average $\Delta V$ and $\Delta I$ values for $n=3$ in Micro90 at $75^{\circ} \mathrm{C}$ with a ramping time of $10 \mathrm{~s}$.

At elevated temperature of $75^{\circ} \mathrm{C}$, the baseline current and the peak value in the electrochemical response curve are much higher than at room temperature, as shown in Fig. 5. This indicates that the degradation mechanism triggered by abrasion is an electrochemical process involving an oxidation reaction whose thermodynamic favorability is enhanced at higher temperatures. Moreover, at a higher temperature, the increased conductivity of a solution could also play a role by making more charged species available to enable faster reaction kinetics on the $\mathrm{TiC}$ domain. Among the three brushing cycles, the current response to the first cycle of brushing is the highest and successive current responses reduce in magnitude. This reduction in current could be attributed to several reasons. It could be due to the hysteresis loosening of the bristles after each brushing motion, or it may be due to a loss of material by abrasion in each brushing cycle, leading to less brushing contact in subsequent brushing cycles. 


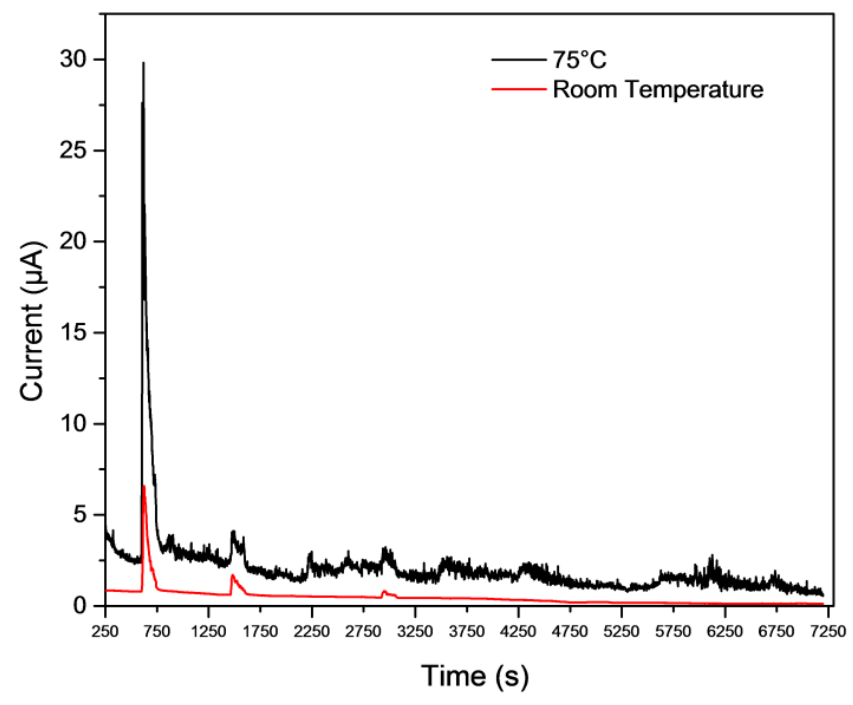

Fig. 5. Effect of temperature on current response to brushing abrasion (3 cycles) in Micro 90.

\subsection{Surface characterization}

SEM images of the sample surfaces after the abrasion test given in Fig. 6 (a-f) show that the abraded samples have been brushed off [37] exhibiting circular 'ploughing' $[38,39,40]$ marks, likely caused by material removal along a curvilinear track. Under a low magnification (at $100 \mathrm{X}$ or lower) these marks appear as concentric rings, consistent with the rotational brushing trajectory (Fig.6 a-c). At a higher magnification, the ploughing marks appear as dark and bright bands. Under closer inspections, these dark bands are formed due to a greater amount of surface wear than brighter bands.

The appearance of these circular bands is affected by the way brush bristles spread on the sample based on the initial contact force between the sample and brush at the beginning of the test. Darker regions show a greater degree of morphological damage and material removal, mostly on the alumina domains than the brighter band region, in which the alumina exhibited much lesser damage. At a 18000X magnification (Fig. 6g), the alumina domain is white and TiC black. In comparison with a pristine sample (Fig. 6h), we clearly see the grain boundary wear and material removal on the alumina domain on the brushed sample (Fig. 6g).

Surface damage as observed in Fig. 6 suggests the susceptibility of the aluminaTiC composite to abrasive wear incurred along with electrochemical activation (Fig. 2) under gentle abrasion condition. Keep in mind that alumina domains appear white 
in higher $\mathrm{kV}$ SEM images from SU6600 and the TiC domain is black, but the contrast reverses in lower $\mathrm{kV}$ SEM images from S4800.

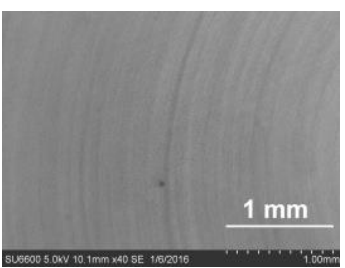

a)

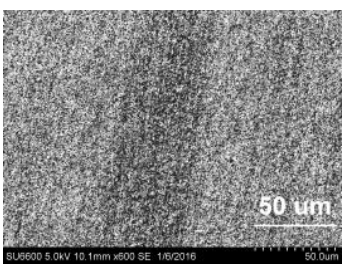

d)

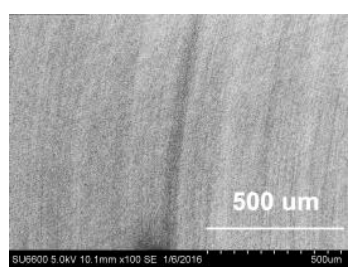

b)

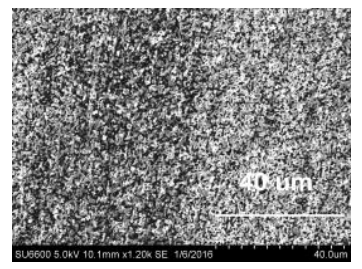

e)

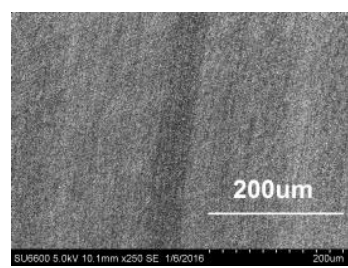

c)

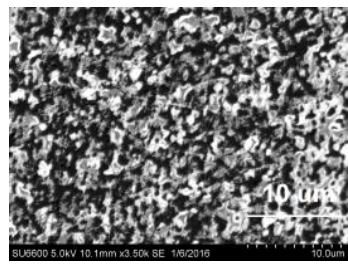

f)

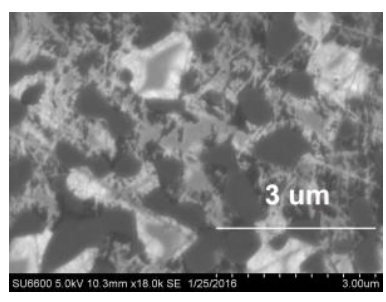

g)

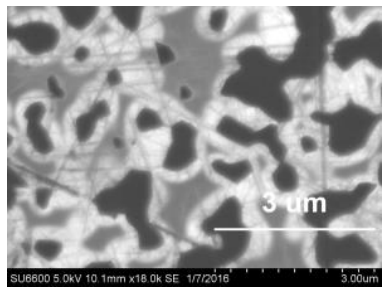

h)

Fig. 6. SEM images (SU6600) of alumina-TiC sample after brushing abrasion in a heated environment at magnifications of a) $40 \mathrm{X}$, b) $100 \mathrm{X}$, c) $250 \mathrm{X}$, d) $600 \mathrm{X}$, e) $1200 \mathrm{X}$, and f) $3500 \mathrm{X}$; $18000 \mathrm{X}$ images of the microstructure of $\mathrm{g}$ ) Brushed and h) Pristine samples.

As seen in Fig.7, the case of a 10 s ramping time which corresponds to the highest current response induces the most severe surface damage than the two other slower cases. High brushing speed causes a similar outcome: more severe surface damage under $1200 \mathrm{rpm}$ than under $500 \mathrm{rpm}$ as shown in (Fig.8). The TiC grain boundaries 
show more wear giving a smeared boundary appearance [37]. As we see in these images, the overall damage is of the same 'microploughing' type. The variation of brushing abrasion parameters is manifested in the severity of damage induced in grain boundary region, with the most severe damage seen for the $10 \mathrm{~s}$ case followed by $40 \mathrm{~s}$ and $70 \mathrm{~s}$ cases. Similarly, a lower maximum speed (500 rpm) causes less damage than a higher maximum speed (1200 rpm). These revealed relationships between electrochemical current response and brushing acceleration and maximum brushing speed and the induced surface damage confirm that the degradation mechanism is an abrasion assisted electrochemical process and the degree of electrochemical interaction of $\mathrm{TiC}$ with the environment depends on the magnitude of parameters controlling the mechanical process of abrasion.

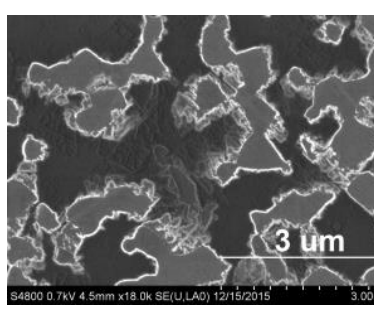

a) $10 \mathrm{~s}$

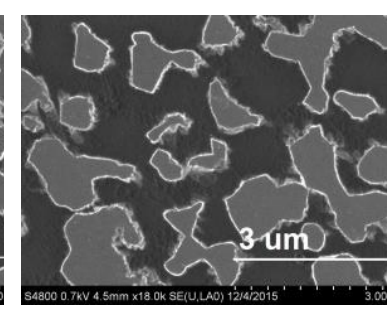

b) $40 \mathrm{~s}$

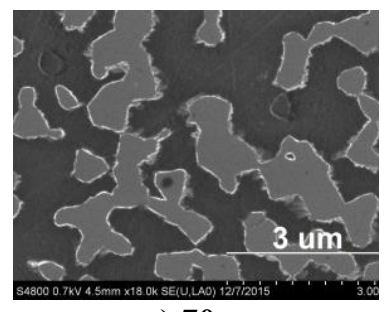

c) $70 \mathrm{~s}$

Fig. 7. Low kV SEM images (S4800) of alumina-TiC showing differences in morphological damage at ramping speeds of a ) $10 \mathrm{~s}$, b) $40 \mathrm{~s}$ and c) $70 \mathrm{~s}$ to maximum brushing speed of 800rpm.

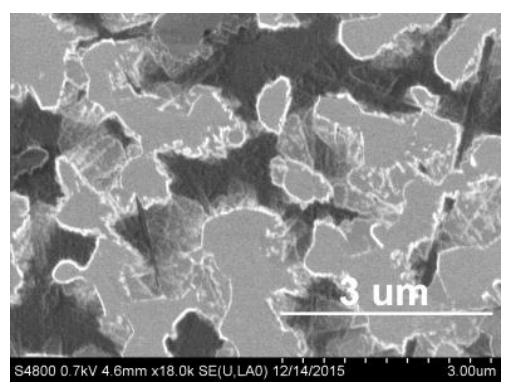

a) $1200 \mathrm{rpm}$

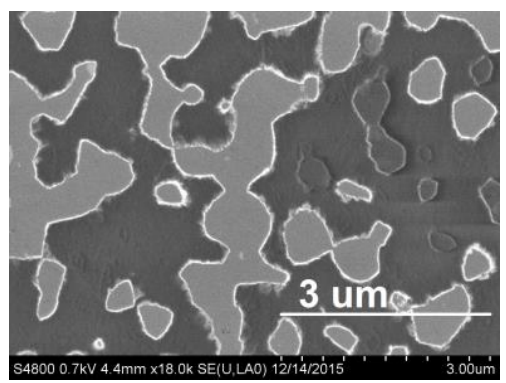

b) $500 \mathrm{rpm}$

Fig. 8. Low kV SEM images (S4800) of alumina-TiC showing differences in morphological damage at different maximum brushing speeds of a) $1200 \mathrm{rpm}$ and b) 500rpm with ramping time of $10 \mathrm{~s}$.

As apparent in Fig.9, grain boundaries are more intact at room temperature and alumina domains show much lesser wear in the room-temperature condition than in 
a heated condition. This morphological damage corresponds well to the current responses obtained (Fig.5) at these at two different temperatures.

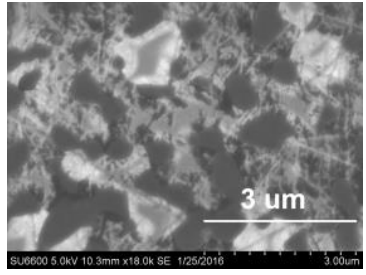

a) Wet- $75^{\circ} \mathrm{C}$

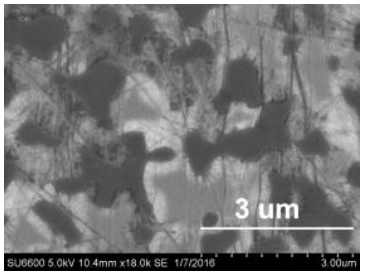

b) Wet- Rt

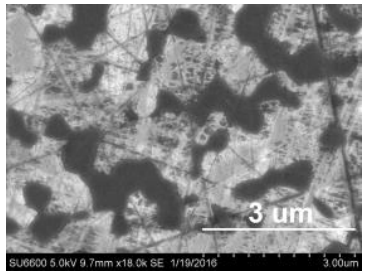

c) Dry-Rt

Fig. 9. Comparison of SEM images (SU6600) of alumina-TiC composite brushed in a) Wet (Micro90) and heated, b) Wet (Micro90) and room temperature(Rt) and c) Dry Rt environment

While comparing the surface of dry brushed samples with wet brushed samples, the material removal of the alumina domain was much less in the dry brushed samples with no damage in the grain boundary region. Damage incurred on alumina domain in a wet environment, especially near grain boundary region could be due to a greater chemical reactivity of alumina to aqueous environment possibly driven by a reaction with water to form hydroxide. At a higher temperature, not only is the rate of electrochemical interactions of $\mathrm{TiC}$ with an aqueous environment higher, the susceptibility of alumina domain to abrasive wear is also increased.

\subsection{Chemical analysis}

XPS analysis of the brushed samples revealed alterations in surface chemistry caused by the oxidative electrochemical process on the TiC domain. Brushed and pristine samples showed the similar elemental composition but their atomic percentages were different (Table 3 ).

Table 3. Atomic percentages of brushed and pristine samples from XPS measurements

\begin{tabular}{|c|l|c|c|c|c|}
\hline & & \multicolumn{4}{|c|}{ Atomic Percent (\%) } \\
\hline Sample/Element & & Ti & C & O & Al \\
\hline Pristine & Average & 3.42 & 38.8433 & 38.96 & 18.77 \\
\hline & Std. Dev & 0.98 & 7.21 & 3.98 & 2.37 \\
\hline Brushed & Average & 7.01 & 28.93 & 47.28 & 16.77 \\
\hline & Std. Dev & 0.88 & 2.02 & 1.23 & 0.66 \\
\hline
\end{tabular}




\begin{tabular}{|c|c|c|c|c|c|}
\hline $\begin{array}{c}\text { Statistical significant } \\
\text { difference }\end{array}$ & $\mathrm{p}<0.05$ & $\mathrm{p}<0.1$ & $\mathrm{p}<0.05$ & $\begin{array}{c}\text { No } \\
\text { difference }\end{array}$ \\
\hline
\end{tabular}

As seen in Table 3, brushed samples show a reduced amount of carbon atoms in the scanned regions along with a higher percentage of titanium and oxygen atoms than the pristine sample. The altered atomic percentages indicate a loss of carbon atoms and acquisition of oxygen on the surface. Further, the regional elemental scans plotted in Fig. 10 for titanium show the relative percentage of the $\mathrm{Ti}_{2 \mathrm{p} 3 / 2}$ bonded to $\mathrm{Ti}$ and the $\mathrm{Ti}_{2 \mathrm{p}} 3 / 2$ bonded to oxygen in $\mathrm{TiO}_{2}$, where a normalized count per second (cps) is obtained with respect to a common peak at approximately $464.4 \mathrm{eV}$ for both samples.

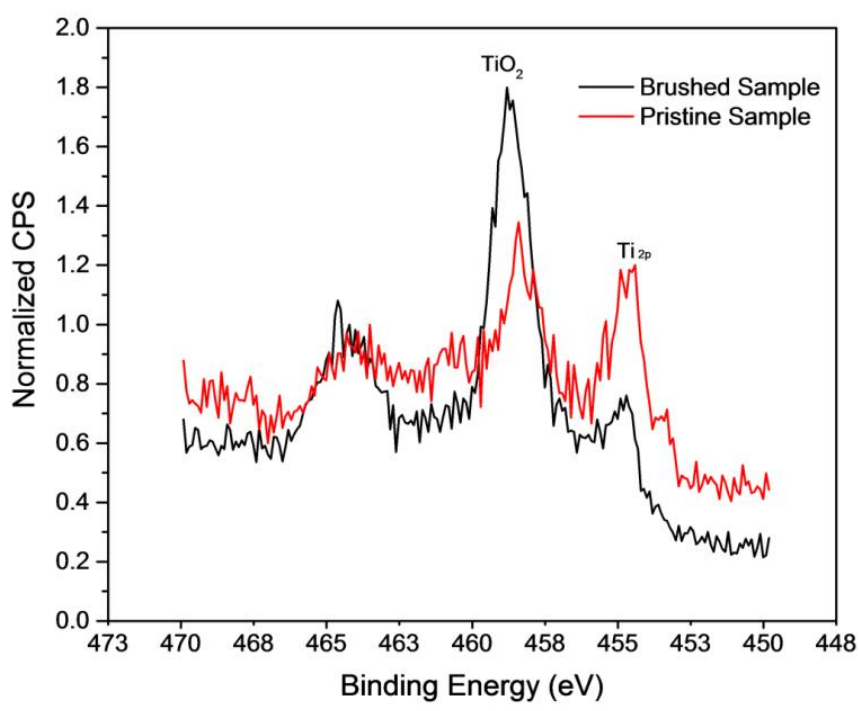

Fig. 10. Elemental scans from XPS analysis for brushed and pristine samples

The pristine sample shows a default $\mathrm{TiO}_{2}$ peak at $458.3 \mathrm{eV}$ with a $\mathrm{Ti}_{2 \mathrm{p} 3 / 2}$ peak at $454 \mathrm{eV}$ representing a Ti-Ti bond. For the brushed sample, the number of Ti bonded atoms to oxygen increased by almost two times as marked by a higher peak at 458.3 eV corresponding to $\mathrm{Ti}_{2 \mathrm{p} 3 / 2} \mathrm{O}_{2}$ formation. Thus, chemical analysis through XPS suggests abrasion induced electrochemical oxidation of $\mathrm{TiC}$ to $\mathrm{TiO}_{2}$ accompanied by a release of some carbon-based product in aqueous environments.

Results of the ICP-MS analysis show the presence of aluminum at $57 \mathrm{ppb}$ for the tested solution and an undetectable level for the untested solution. For titanium, the amount is below the detection limits for both solutions. Higher aluminum concentrations in the tested solutions indicate that even with gentle abrasion the composite will release wear particles into the test solution, though the precise chemical state, e.g., whether alumina or aluminum hydroxide, is unknown. These 
particles may have been immediately swept away from sample surface and brushed along the surface. If the loose particles are alumina, they could result in ploughing of the sample surface.

\subsection{Electrochemical Impedance data analysis}

Electrochemical impedance spectroscopy data can provide crucial information about the state of the oxide film at the composite-solution interface. An oxide film formed on a surface often exhibits resistive (frequency-independent) and capacitive (frequency dependent) behavior. The Nyquist plot is an effective way to characterize the charge transfer processes. In a Nyquist plot (often a semicircle), fast kinetic controlled reactions are represented in a high frequency region at the left end, and slow diffusion and mass transfer controlled reactions are captured in a low frequency region at the right end, as depicted in Fig.11b. With the equivalent Randles circuit shown in Fig.11a, containing a constant phase element (CPE), charge transfer resistance of oxide films $\left(R_{c t}\right)$ and solution resistance $\left(R_{s}\right)$, we can determine the various parameters through statistical fitting of the circuit model to the Nyquist plots. Here $R_{\mathrm{s}}$ measures the resistance present in solution between the reference electrode and working electrode, which is affected by ionic concentration, temperature, type of ions and area of electrode. $\mathrm{R}_{\mathrm{ct}}$ is the resistance of the oxide film and electrode-electrolyte interface to charge transfer, and it varies with the type of reactions, conditions of oxide film (compact or porous with defects), temperature, electrode potential and concentration of reactant species. CPE is a non-ideal representation of capacitive behavior of an electrical double layer on an electrodeelectrolyte interface [41]. For analyzing EIS data, the alumina domain is considered not to be participating in the charge transfer processes during electrochemical interactions. 
a)

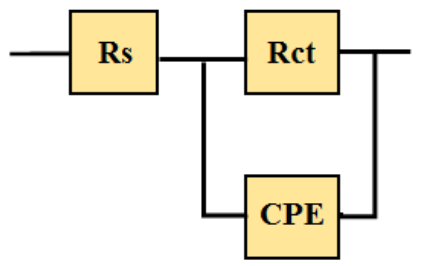

b)

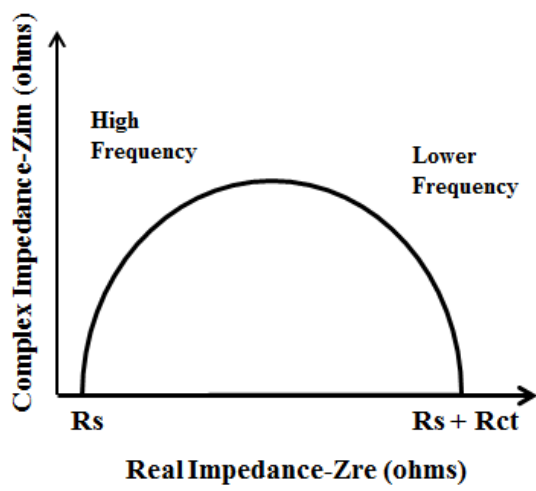

Fig. 11. a) Equivalent circuit for Randles cell and b) Corresponding Nyquist plot.

$R_{c t}$ values before and after each test run in experimental conditions mentioned in Table 2 were obtained by fitting of the equivalent circuit model to the inner semicircle (high frequency region) of the Nyquist data obtained (Fig.12). Frequency dependent behavior of the phase between the applied input and measured output signal and impedance at electrode interface in Bode plot (not shown) was marked by a one time constant, which is representative of a single R-C component like equivalent circuit for a Randles cell. 

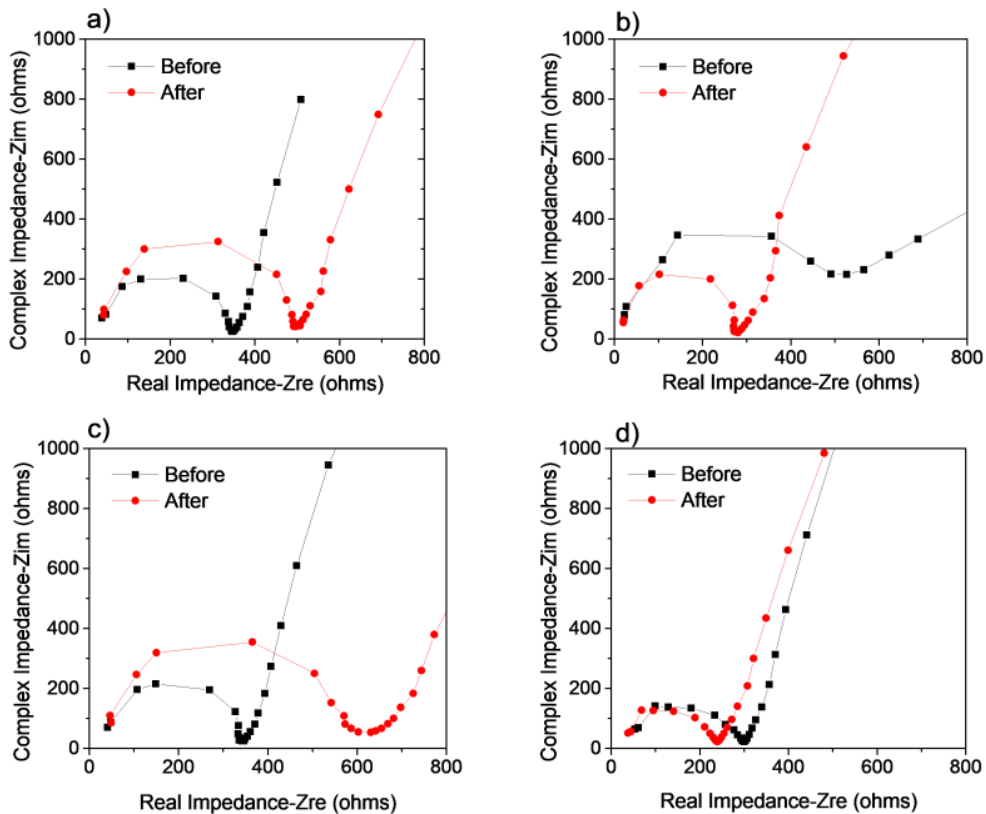

Fig. 12. Nyquist plot obtained before and after each experiment. a) Anodic biasing (250mV vs SCE), b) Cathodic biasing (-250mVvs SCE), c) No Brushing-No Biasing, and d) Brushing-No Biasing.

After anodic biasing $\left(250 \mathrm{mV}\right.$ vs SCE), the $\mathrm{R}_{\mathrm{ct}}$ value increased, indicating formation of a stable and compact barrier to charge transfer processes verifying the tendency of the $\mathrm{TiC}$ domain to form $\mathrm{TiO}_{2}$ in an aqueous environment. Similar behavior was observed when the sample was just exposed to the test solution under a no-biasing condition without brushing abrasion. However, after cathodic biasing $\left(-250 \mathrm{mV}\right.$ vs SCE), $\mathrm{R}_{\mathrm{ct}}$ values reduced. Cathodic potentials are known to deteriorate the stability of the $\mathrm{TiO}_{2}$ film [3] that results in an increased amount of electrochemical interaction of $\mathrm{TiC}$ with the environment and enhanced charge transfer rate at the interface. The brushing with a no-biasing condition, which favors oxide film formation under undisturbed conditions, produces reduced $R_{c t}$ as seen in Fig.13 after brushing abrasion. 


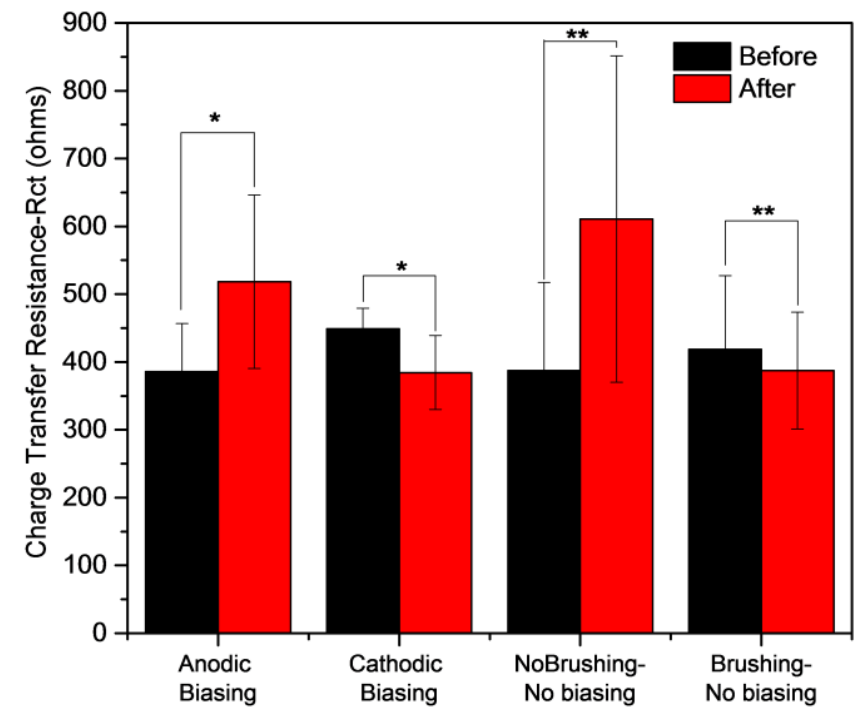

Fig. 13. Charge transfer resistance $R_{c t}$ values for composite sample before and after each experimental test condition in Micro90 at $75^{\circ} \mathrm{C}(*$ : significant difference $(p<0.05) \& * *$ : significant difference $(p \leq 0.1)$.

\subsection{Understanding the degradation mechanism of alumina-TiC composite}

In the current study, the alumina-TiC composite underwent 'gentle abrasion' and the fundamental degradation mechanism can be categorized as triboelectrochemical wear. The oxidative current response and ploughing damage on the composite surface are all indicators of tribo-electrochemical wear induced by brushing abrasion. There is no visible wear on TiC grains due to its higher hardness [22] and wear resistance. However, TiC likely undergoes oxidation [30,31] to form $\mathrm{TiO}_{2}$ and $\mathrm{CO}_{2}$ as per the following reaction:

$$
\mathrm{TiC}+4 \mathrm{H}_{2} \mathrm{O} \rightarrow \mathrm{TiO}_{2}+\mathrm{CO}_{2}(\mathrm{aq})+8 \mathrm{H}^{+}(\mathrm{aq})+8 \mathrm{e}^{-}
$$

Upon contact with an aqueous environment and when left undisturbed, a passive oxide layer would form on $\mathrm{TiC}$, as the $\mathrm{R}_{\mathrm{ct}}$ value plotted in Fig.13 indicates. The onset of brushing could lead to the removal of micro-asperities on the alumina domain along with the rupture of a passive layer on $\mathrm{TiC}$ thus activating a burst of 
electrochemical oxidative processes on conductive TiC as shown in Eq. (1). While the $\mathrm{TiC}$ domains would repassivate and form $\mathrm{TiO}_{2}$, the ongoing brushing would keep the electrochemical interactions of $\mathrm{TiC}$ active due to ongoing abrasion of any possible oxide layer formed. Under this situation, a stable oxide layer can hardly exist. Aside from $\mathrm{TiO}_{2}$ formation, the reduction in carbon percentage in XPS results point toward the release of carbon atoms into the solution to form $\mathrm{CO}_{2}$ as per Eq.1 due to oxidation and replacement of carbon by oxygen in the TiC crystal lattices.

In general, gentle wear process like the one performed in this study would cause less damage [23]. Through qualitative comparison of the surface morphology of abraded samples under different environment conditions, it is clear that the alumina domain showed a greater amount of material 'chipped away' in a wet and heated environment than at wet room temperature and dry brushing conditions. Alumina experiences tribological and chemical degradation. The hydrophilic nature of alumina causes it to readily hydrate to aluminum hydroxide when in contact with water and this reaction is thermodynamically more prevalent at higher temperatures $[42,43]$. Strong reactivity to water and resulting greater surface plasticity of alumina in a wet environment makes it more susceptible to wear in a wet environment than dry conditions [23]. During abrasion, the hydroxide formed that may not be adherent is abraded away along with chipping of brittle alumina domain as supported by ICP-MS analysis. The low stress during brushing abrasion leaves released debris unconstrained [44] and they could be immediately swept away from the sample surface and brushed along creating circular trajectories further abrading the alumina matrix. Thus, debilitated wear resistance of the alumina domain in a heated aqueous alkaline environment plays a crucial role in the degradation of the composite.

Relating this understanding to the evidence from altered charge transfer resistance, the wear debris released also interrupts the passive $\mathrm{TiO}_{2}$ layer formation on the TiC domain during continuous brushing. It is important to note that the electrochemical current response may have negligible or no contributions from alumina even though it could be chemically reacting with an aqueous environment. However, XPS chemical analysis of the brushed sample for two hours does show traces of $\mathrm{TiO}_{2}$. This indicates that prolonged brushing and exposure to the solution does result in $\mathrm{TiO}_{2}$ formation in worn out regions, however, its structure may not be uniform and compact to offer any resistance to oxidative charge transfer processes because of brushing abrasion. A similar reduction in $\mathrm{R}_{\mathrm{ct}}$ after abrasion due to damage in the protective layer was observed by others $[45,46]$.

\section{Conclusions}

This study has shed new insights into the interplay of abrasion and electrochemical degradation of alumina-TiC ceramic composites in an aqueous environment. An oxidative electrochemical process on $\mathrm{TiC}$ is activated by brushing abrasion while alumina also undergoes abrasive wear. It is established in this chapter that: 
- Brushing abrasion causes electrochemical activation of alumina-TiC composites, forming $\mathrm{TiO}_{2}$ on the composite surface due to oxidation of $\mathrm{TiC}$ in an aqueous environment.

- Abrasive damage occurs near the grain boundaries with traces of "microploughing" on alumina domains.

- Electrochemical response to brushing abrasion and the corresponding surface damage are affected by abrasion parameters like acceleration and speed of the abrasion motion and the temperature of test environments.

- An elevated temperature enhances thermodynamic favorability and reaction rate of TiC oxidation and enables faster charge transfer.

- A wet and heated environment increases susceptibility of abrasion damage in alumina domains in comparison with a dry condition.

- Wear debris released from alumina abrasion may hinder the formation of a protective $\mathrm{TiO}_{2}$ film.

It is crucial to consider that abrasion mechanisms employed in the study are gentle and under extremely low load conditions and such mechanically assisted electrochemical degradation mechanism is certainly to be aggravated under a greater load in aggressive ionic environments present in the biological milieu.

Acknowledgments The authors would like to acknowledge the support from the Department of Bioengineering and the Institute for Biological Interfaces of Engineering at Clemson University. Funding support for this work is provided by a storage media company through a research agreement with Clemson University under contract number 146422 and by the Institute for Biological Interfaces of Engineering at Clemson University.

\section{References}

1. Jazrawi, L. M., Kummer, F. J.,Di Cesare, P. E. (1998). Hard bearing surfaces in total hip arthroplasty. American journal of orthopedics (Belle Mead, NJ), 27(4), 283-292.

2. Bard, A. J., Faulkner, L. R., Leddy, J., \& Zoski, C. G. (1980). Electrochemical methods: fundamentals and applications (Vol. 2). New York: Wiley, pp 44-82

3. Gilbert, J. L., Mali, S. A. (2012). Medical implant corrosion: Electrochemistry at metallic biomaterial surfaces. In Degradation of Implant Materials (pp. 1-28). Springer New York.

4. Royhman, D., Patel, M., Runa, M. J. et al (2016). Fretting-corrosion behavior in hip implant modular junctions: the influence of friction energy and $\mathrm{pH}$ variation. Journal of the Mechanical Behavior of Biomedical Materials, 62, 570-587.

5. Swaminathan, V., Gilbert, J. L. (2012). Fretting corrosion of CoCrMo and Ti6Al4V interfaces. Biomaterials, 33(22), 5487-5503.

6. Cooper, H. J., Della Valle, C. J., Berger et al (2012). Corrosion at the head-neck taper as a cause for adverse local tissue reactions after total hip arthroplasty. The Journal of Bone \& Joint Surgery, 94(18), 1655-1661. 
7. Cooper, H. J., Urban, R. M., Wixson, R. L. et al. (2013). Adverse local tissue reaction arising from corrosion at the femoral neck-body junction in a dual-taper stem with a cobalt-chromium modular neck. J Bone Joint Surg Am, 95(10), 865872.

8. Begin-Colin, S., Mocellin, A., Stebut, J. V. et al (1998). Alumina and Alumina-TiN wear resistance in a simulated biological environment. Journal of materials science, 33(11), 2837-2843.

9. Lashneva, V. V., Kryuchkov, Y. N.,Sokhan, S. V. (1998). Bioceramics based on aluminum oxide. Glass and ceramics, 55(11), 357-359.

10. Garino, J., Rahaman, M. N., Bal, B. S. (2006, December). The reliability of modern alumina bearings in total hip arthroplasty. In Seminars in Arthroplasty (Vol. 17, No. 3, pp. 113-119). WB Saunders.

11. Skinner, H. B. (1999). Ceramic bearing surfaces. Clinical orthopaedics and related research, 369, 83-91.

12. Willmann, G. (2000). Ceramic femoral head retrieval data. Clinical orthopaedics and related research, 379, 22-28.

13. Boutin, P., Christel, P., Dorlot, J. M. et al (1988). The use of dense aluminaalumina ceramic combination in total hip replacement. Journal of biomedical materials research, 22(12), 1203-1232.

14. Nine, M. J., Choudhury, D., Hee, A. C. et al (2014). Wear debris characterization and corresponding biological response: artificial hip and knee joints. Materials, 7(2), 980-1016.

15. Mittelmeier, H., Heisel, J. (1992). Sixteen-years' experience with ceramic hip prostheses. Clinical orthopaedics and related research, 282, 64-72.

16. Jianxin, D., Zeliang, D., Jun, Z. et al (2006). Unlubricated friction and wear behaviors of various alumina-based ceramic composites against cemented carbide. Ceramics international, 32(5), 499-507.

17. Fei, Y. H., Huang, C. Z., Liu, H. L. et al (2014). Mechanical properties of Al $2 \mathrm{O}$ 3-TiC-TiN ceramic tool materials. Ceramics International, 40(7), 10205-10209.

18. Guu, Y. Y., Lin, J. F.,Ai, C. F. (1997). The tribological characteristics of titanium nitride, titanium carbonitride and titanium carbide coatings. Thin solid films, 302(12), $193-200$.

19. Lee, S. W., Morillo, C., Lira-Olivares, J. et al (2003). Tribological and microstructural analysis of $\mathrm{Al} 2 \mathrm{O} 3 / \mathrm{TiO} 2$ nanocomposites to use in the femoral head of hip replacement. Wear, 255(7), 1040-1044

20. Cai, K. F., McLachlan, D. S., Axen, N. et al. (2002). Preparation, microstructures and properties of Al2O3-TiC composites. Ceramics International, 28(2), 217-222.

21. Brama, M., Rhodes, N., Hunt, J. et al (2007). Effect of titanium carbide coating on the osseointegration response in vitro and in vivo. Biomaterials, 28(4), 595-608.

22. Shackelford, J. F., Han, Y. H., Kim, S., \& Kwon, S. H. (2016). CRC materials science and engineering handbook. CRC press.

23. Sahoo, P.,Davim, J. P. (2013). Tribology of Ceramics and Ceramic Matrix Composites. In Tribology for Scientists and Engineers (pp. 211-231). Springer New York.

24. Oda, K.,Yoshio, T. (1997). Hydrothermal corrosion of alumina ceramics. Journal of the American Ceramic Society, 80(12), 3233-3236.

25. Lauwers, B., Kruth, J. P., Liu, W., Eeraerts, W., Schacht, B., \& Bleys, P. (2004). Investigation of material removal mechanisms in EDM of composite ceramic materials. Journal of Materials Processing Technology, 149(1), 347-352.

26. Landfried, R., Kern, F., Burger, W., Leonhardt, W., \& Gadow, R. (2013). Development of Electrical Discharge Machinable ZTA Ceramics with 24 vol\% of 
TiC, TiN, TiCN, TiB2 and WC as Electrically Conductive Phase. International Journal of Applied Ceramic Technology, 10(3), 509-518.

27. Avasarala, B., \& Haldar, P. (2010). Electrochemical oxidation behavior of titanium nitride based electrocatalysts under PEM fuel cell conditions. Electrochimica Acta, 55(28), 9024-9034.

28. Meijs, S., Fjorback, M., Jensen, C., Sørensen, S., Rechendorff, K., \& Rijkhoff, N. J. (2015). Electrochemical properties of titanium nitride nerve stimulation electrodes: an in vitro and in vivo study. Frontiers in neuroscience, 9.

29. Zhang, L., Koka, R. V. (1998). A study on the oxidation and carbon diffusion of TiC in alumina-titanium carbide ceramics using XPS and Raman spectroscopy. Materials chemistry and physics, 57(1), 23-32.

30. Cowling, R. D.,Hintermann, H. E. (1970). The corrosion of titanium carbide. Journal of The Electrochemical Society, 117(11), 1447-1449.

31. Cowling, R. D., Hintermann, H. E. (1971). The anodic oxidation of titanium carbide. Journal of The Electrochemical Society, 118(12), 1912-1916.

32. Kiran, V., Srinivasu, K., Sampath, S. (2013). Morphology dependent oxygen reduction activity of titanium carbide: bulk vs. nanowires. Physical Chemistry Chemical Physics, 15(22), 8744-8751.

33. Ramirez, A. G., Kelly, M. A., Strom, B. D. et al (1996). Carbon-coated sliders and their effect on carbon oxidation wear. Tribology transactions, 39(3), 710-714.

34. Contu, F., Elsener, B., Böhni, H. (2005). Corrosion behaviour of CoCrMo implant alloy during fretting in bovine serum. Corrosion Science, 47(8), 1863-1875.

35. Barril, S., Debaud, N., Mischler, S., et al (2002). A tribo-electrochemical apparatus for in vitro investigation of fretting-corrosion of metallic implant materials. Wear, 252(9-10), 744-754

36. Bratu, F., Benea, L., Celis, J. P. (2007). Tribocorrosion behaviour of Ni-SiC composite coatings under lubricated conditions. Surface and Coatings Technology, 201(16), 6940-6946

37. Jianxin, D., Tongkun, C., Zeliang, D. et al (2006). Tribological behaviors of hotpressed $\mathrm{Al} 2 \mathrm{O}$ 3/TiC ceramic composites with the additions of $\mathrm{CaF} 2$ solid lubricants. Journal of the European Ceramic Society, 26(8), 1317-1323.

38. Jahanmir, S. (2002). Wear transitions and tribochemical reactions in ceramics. Proceedings of the Institution of Mechanical Engineers, Part J: Journal of Engineering Tribology, 216(6), 371-385.

39. Yingjie, L., Xingui, B., Keqiang, C. (1985). A study on the formation of wear debris during abrasion. Tribology international, 18(2), 107-111.

40. Lee, G. Y., Dharan, C. K. H., Ritchie, R. O. (2002). A physically-based abrasive wear model for composite materials. Wear, 252(3), 322-331.

41. Instruments, G. Basics of Electrochemical Impedance Spectroscopy. 2006. Gamry Instruments: 20Primer, 202006.

42. Gee, M. G. (1992). The formation of aluminium hydroxide in the sliding wear of alumina. Wear, 153(1), 201-227.

43. Gates, R. S., Hsu, M.,Klaus, E. E. (1989). Tribochemical mechanism of alumina with water. Tribology transactions, 32(3), 357-363.

44. Gates, J. D. (1998). Two-body and three-body abrasion: a critical discussion. Wear, 214(1), 139-146.

45. Azzi, M., Szpunar, J. A. (2007). Tribo-electrochemical technique for studying tribocorrosion -behavior of biomaterials. Biomolecular engineering, 24(5), 443446.

46. Vitry, V., Sens, A., Kanta, A. F.et al (2012). Wear and corrosion resistance of heat treated and as-plated Duplex NiP/NiB coatings on 2024 aluminum alloys. Surface and Coatings Technology, 206(16), 3421-3427. 
